J. Gen. Appl. Microbiol.

Vol. 5 , No. 4,1960

\title{
STUDIES ON THE CLASSIFICATION OF STREPTO- MYCETES, PART XII* THE MICROSCOPIC MORPHOLOGY AND OTHER CHARACTERISTICS OF STREPTOMYCES HYGROSCOPICUS AND MORPHOLOGICALLY RELATED STRAINS
}

\author{
RYOSAKU NOMI \\ Faculty of Engineering, University of Hiroshima \\ Received for publication, September 20, 1959
}

Streptomyces are, as it is often pointed out, morphologically well differentiated and similar to fungi. In 1919, DreChSLER (1) reported considerably detailed morphological characteristics of Actinomyces, but the study received few attention. Since then, Waksman et al. (2), Krassilnikov (3), Baldacci et al. (4) and Gause proposed taxonomic system which attached great importance to physiological and cultural properties and which more or less made light of morphological properties. In 1958, Pridham et al. (5) proposed the subdivision of the genus Streptomyces into several morphological sections. They have successfully adopted morphology of sporiferous part as a taxonomic criterion. Recently, EтtLinger et al. (6) reported another taxonomic system in which morphological properties were adopted as important criteria.

Now, the author considers that, in morphology of Streptomyces, not only sporiferous part, but also other parts of aerial mycelia have morphological characteristics distinctly enough to be adopted as taxonomic criteria.

Tresner and BAckus ( 7 ) investigated various morphological and physiological characteristics of eighteen strains of Streptomyces hygroscopicus and their related species. They concluded that the most constant characteristics in Streptomyces hygroscopicus were spore color en masse, tightly wounded coils of the spore-bearing hyphae arisen as short side branches of main hyphae and the black hygroscopic areas developed on colonies. They also pointed out that other various cultural and physiological features were variable.

The author $(8 \sim 15)$ has already reported on the various types of morphology in Streptomyces and has pointed out that microscopic morphology is a stable characteristics. In this paper, the author intends to report on the detailed microscopic morphological characteristics of Streptomyces hygroscopicus and their morphologically related strains. The morphological characteristics presented in this paper is a new morphological type in Streptomyces which differs from the other morphological types reported in the previous papers.

* Parts I-XI have been published in J. Ferment. Tech., Japan, 31 (1953) 32 (1954) and $\mathbf{3 3}$ (1955). 


\section{EXPERIMENTS AND RESULTS}

\section{Strains employed in this study}

The strains employed are Streptomyces hygroscopicus (JENSEN) WaKSman et HenRICI HUT 6026 [ $\leftarrow$ IFO $3192 \leftarrow$ CBS (Westerdijk)], Streptomyces violaceo-

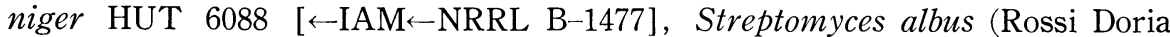
emend. Krainsky) WaKsman HUT 6047 [ (A. J. Kluyver)] and six other strains which were isolated from the soil at this laboratory. While these six strains are called by strain number, they may be identified with already published type cultures. But in author's collection of type cultures no same cultures as any of these six strains can be found.

Nowadays, specification of species is usually described according mainly to cultural and physiological characteristics of strain, while the morphological characteristics are not always described in detail. But the author considers it desirable to adopt more than ever morphological characteristics as taxonomic criteria. Accordingly, when the author wishes to identify some strain with an already published type strain, he must study the detailed morphological characteristics of the type strain. Because, when the identification was made using only the cultural and physiological criteria and not using detailed morphological criteria, there may be possibility to identify the isolated strain with a type strain, though they are apparently of morphological difference. Such case can be instanced in the author's collection of type cultures itself. Therefore, untill the author obtains the type strain which, both morphologically and physiologically, is considered to be of the same species with the author's unnamed strain, the author postpones the denomination of above six strains.

\section{Method of microscopic observation}

The detailed observation of microscopic morphology was carried out using cover culture method (16) together with plate observation method. The medium used is Bennett's agar or modified Bennett's agar. In Bennett's agar glucose is used as carbon source, but glucose is not always an ideal carbon source for all strains to produce the aerial mycelium. As will be made clear later in this paper, the c-source for good production of aerial mycelium varies as to strains employed. In morphological study the suitable c-source need to be used. Usually glycerol, xylose, glucose, fructose, mannitol, sucrose, maltose, lactose and starch are examined. In modified Bennett's agar c-source is favourably substituted for glucose in Bennett's agar. If necessary, the concentration of c-source is modified to desired consistency such as half or three times as much as original concentration. When the quality or quantity of c-source is inadequate, the microscopic characteristics show the incompletely grown morphology whose subsequent growth has been suspended. 


\section{Cultural characteristics}

The slant cultural features on Czapek-Dox agar, Krainsky agar, Bennett agar and modified Bennett agar, and starch hydrolysis test and behavior on skim-milk and nitrate reduction test of each strain were studied and recorded. Incubation was carried out for fourteen days at $28-30^{\circ} \mathrm{C}$, with the exception of seven days incubation in starch hydrolysis test. The data will be given at the end of this paper, together with some special morphological features of each strain, under "Description of Strain".

It is almost a tradition among mycologist that in classification of microorganism the complete morphological growth phase should be made to research first of all and with as much exertion as possible. And the color of aerial mycelium should always be referred to in connection with the morphology of sexual organ. Now, the author considers that, in Streptomyces, great importance is to be given to the color of aerial mycelium when the full morphology has been formed and the spores have matured. In Streptomyces, even if the full morphology has been formed, the color of aerial mycelium may vary in some degree when the cultural medium is changed and often even on a slant culture. However, the variation of color in those cases occurs within some certain range of a color series. Therefore, in describing the color of culture, it is desirable to express in terms of some width of color. Moreover, if any other special color occurred at some period of growth or on some cultural medium used, the color should be expressed as an instance of special reference.

\section{Microscopic morphology}

Morphological characteristics of each strain employed are not just the same, having, as they do, some special features which are different from each other. It is described at the end of this paper together with the cultural characteristics. On the other hand, each strain has general morphological features which are common to all strains employed and which apparently distinguish themselves from other types of morphology in Streptomyces, the latter has already been reported and will be reported in succeeding papers. In this section, reference is solely made to the general morphology of the strains employed.

General morphology of the strains employed: The aerial mycelia are well developed but the branching system is not simple. The aerial mycelia sometimes take the shape of comparatively long straight or long wavy and sometimes short straight or short wavy, and they branch in monopodial alternate, opposite and verticillate forms especially in their young stage of growth. But in accordance with their growth the branching system occasionally becomes to seem irregular. It is because, when the aerial mycelia have well developed and repeatedly branched, the degree of development and the direction of branches are not uniform and sometimes the direction of branches at distal parts is just opposite to it at starting part. Furthermore, branching form of aerial mycelia sometimes seems like sympodial system. 
And on the whole, there are no main axial mycelia, and repeatedly diverged branches are still generally axial mycelia. Occasionally, under low microscopic magnification, they seem cluster-like or coelicolortype (8)-like. Now, what is significant and noticeable is that they do not break into oidiospores. Sporiferous parts are, indeed, side branches which arise from those various axial mycelia. Sometimes long aerial mycelium earths on the medium, from which point vegetative mycelium grows on again. As the result of those growth system mentioned, no regularity is often observed in branching system of aerial mycelia after good growth, and the microscopic appearance of them occasionally appears net-like at a glance.

The monopodially or irregularly branched axial mycelia give rise to side branches which branch on further in various degree, i. e. from single to much diverged and complicated branches. The side branches develop into spirals or wavy hyphae in terminal filaments which break into oidiospores. The number of branches in a side branch is not constant in a strain and length of terminal spirals or wavy hyphae also differs from strain to strain and so it does within a strain. When a side branch is thickly branched, its morphology, in itself, is similar to the morphological type of Streptomyces coelicolor et al. $(8,9,13)$

In spirals, the number of winding, loose or compact, and dimension of diameter are about constant in a strain within certain range, which is different from strain to strain. The direction of turn in spiral, namely dextrorse or sinistrorse, is exclusively constant in a strain. In all strains employed in this study, it is sinistrorse. The direction of turn is decided by tracing the spiral in this direction from far to near. (In author's previous papers, $(8 \sim 14)$ the direction of turn was decided by tracing in contrary direction to it in this paper. But, as the author has found the previous method botanically mistaken, it is amended from this paper on.) In terminal spiral parts of some strains employed, a sympodially branched chain of a few spirals is sometimes observed.

Oidiospores are of various shape. In Streptomyces, the shape of oidiospores is either sphere, ellipsoid, short rod or rod. The shape of oidiospores in a strain is not only of a kind of shape, but principally contains various shapes. In spiral's coiled parts or short wavy sporiferous parts, the shape of oidiospores is generally sphere to ellipsoid (or short rectangle), and in long straight sporiferous parts, it generally contains many longer shapes, i.e. from ellipsoid to short rod to rod. As the shape of oidiospores is correlative with morphology of sporiferous parts, the shape of spores is not always significant in taxonomic criterion.

In this morphological type, there is often contained some transitional types which is different from the above-mentioned morphology. On the colonial part bearing long aerial mycelia in abundance, the morphology resembles that of Streptomyces sp. No. 477 (14) reported in the previous paper. In the latter, long straight aerial mycelia project radially, branching in monopodial alternate, opposite and verticillate forms, and the branches follow 
the same branching pattern. The long straight aerial mycelia do not break into oidiospores but give rise to short side branches, which branch, on further, in various degree, i.e. from single to much diverged branches. The terminal filaments of these side branches form spirals or short wavy filaments and sporulate.

On the colonial part bearing no long aerial mycelia, the morphology resembles that of Streptomyces coelicolor et al. $(8,9,13)$ reported in the previous papers. In the latter, the aerial mycelia are comparatively straight or wavy in shape and branch in monopodial alternate, opposite and verticillate forms, and the branches follow the same branching pattern in various degree. The terminal filaments give rise to spirals or short wavy filaments and sporulate.

In the morphological type presented in this paper, those two transitional morphology and the above-mentioned irregularly branched morphology are simultaneously included in various ratio and such ratio differs from strain to strain.

Now, therefore, the occurrence of morphological segregation may be expected in this morphological type; for instance, some strain studied may morphologically segregate to Streptomyces sp. No. 477 type and Streptomyces coelicolor type. But the author's experiment has denied the segregative possibility, to which some experimental data will be given in the following paper.

The appended photographs will be made available for understanding general morphology of the strains studied.

\section{DESCRIPTION OF STRAIN}

For experimental items, see the sections of "Cultural characteristics" and "Microscopic morphology". In the following description, the abbreviation, (A), (V), (R) and (P), mean aerial mycelium, vegetative mycelium, reverse of colony and soluble pigment, respectively. On the aerial mycelium, the degree of development is expressed in terms of five grades; non, slight, moderate, fairly good and good. Italic color name is quoted from RIDGWAY's Color Standards and Nomenclature 1912. In modified Bennett's agar, favourable carbon sources are shown in round brackets. Although the color of aerial mycelium on the morphologically unfavourable cultural medium is not necessarily significant in taxonomic study, all the cases of color will be referred to when aerial mycelium is produced for the sake of comparison with other experimental data.

In the following description the name of slant cultural medium is curtailed "agar".

\section{Streptomyces hygroscopicus HUT 6026}

I. Long axial mycelia are fairly developed and either straight or a 
little flexous. They are much longer than side branches. Sometimes axial mycelia are short in length.

Side branches are single to about three step' branching with 5 to 8 terminal hyphae in many cases, but occasionally more abundantly branched. When abundantly branched, it is impossible to distinguish them from short axial mycelium with short side branches. Side branches arise thickly from axial mycelium. Terminal hyphae in side branches are short in length.

Spiral: sinistrorse, very compact (each ring of spiral is adhered to compact (each ring not adhered), 1 to 6 turns, 2.5 to $5 \mu$ in diameter, mostly cylindrical and sometimes snail-like. Length of spiral (including spiralphore) is 3 to $12.5 \mu$. Spore: sphere to ellipsoid to rectangle, 1.0 to 1.4 by 1.0 to $1.8 \mu$ in diameter.

Characteristic moist and dark brownish hygroscopic areas develop on colonies.

II. Czapek: (A) fairly good; white. (R) brownish white. (P) almost non. Krainsky: (A) slight; white to a few patches of Hair Brown. (R) yellowish white. (P) non. Bennett: (A) slight; white to a few patches of brownish black. (R) pale yellow. (P) non. Mod. Bennett (xylose and maltose): (A) good; Drab-Gray, Light Drab and Mouse Gray $\rightarrow$ Fuscous $\rightarrow$ Fuscous-Black, Seal Brown or Blackish Brown (1) patches when self-moistened. (R) pale yellow. (P) non.

Starch: Hydrolysis positive. Milk: No coagulation; peptonization positive. Nitrate: Reduction very weak or negative.

\section{Streptomyces violaceoniger HUT 6088}

I. Almost the same as S. hygroscopicus HUT 6026. In comparison, long axial mycelia are small in number and turns of spiral are few.

Spiral: sinistrorse, very compact to compact, 1 to 4 turns, 2.5 to $5 \mu$ in diameter, almost cylindrical. Length of spiral is 3 to $9.5 \mu$.

Hygroscopic areas develop and are almost black.

II. Czapek: (A) non. (V) colorless to slightly yellow. (R) pale yellowish brown. (P) pale brownish yellow. Krainsky: (A) non. (V) pale yellow to yellow. (R) pale yellow to yellow. (P) almost non. Bennett: (A) slight: white. (R) yellowish orange. (P) almost non. Mod. Bennett (xylose): (A) fairly good; Smoke Gray to Pale Mouse Gray $\rightarrow$ Pale (or Light) Purplish Gray, Pale Neutral Gray $\rightarrow$ moist black patches when self-moistened. (R) yellow to yellowish brown. (P) almost non.

Starch: Hydrolysis positive. Milk: No coagulation; peptonization positive. Nitrate: Reduction negative.

\section{Storeptomyces albus HUT 6047}

I. In early stage of growth, the branching system is almost similar to $S$. coelicolor et al. But in its stage of development, the non-sporulated axial filaments become much longer in length to exceed that of side branches, their shape being not straight but more or.less flexous. 
The terminal hyphae in side branches are longer than those of the above two strains.

Spiral: sinistrorse, very compact to compact, 1 to 5 turns, 3 to $6 \mu$ in diameter. Length of spiral is 4 to $18 \mu$. Spore: ellipsoid to short rod, 0.6 to 0.9 by 0.7 to $1.4 \mu$ in diameter.

II. Czapek: no growth. Krainsky: (A) moderate; white. (R) white to yellowish white. (P) non. Bennett: (A) good; white. (R) yellowish brown. (P) pale yellow. Mod. Bennett (all 9 c-sources):(A) good; white. (R) yellow or yellowish brown. (P) non to pale yellow.

Starch: No growth on ammonium sulfate medium. On starch-Bennet. agar and starch-Krainsky agar plate, starch is not hydrolyzed. Milk: No coagulation; peptonization positive; red soluble pigment. Nitrate: Reduction negative or very weak.

Streptomyces sp. No. 201

I. About the same as $S$. albus HUT 6047.

Spiral: sinistrorse, very compact, 1 to 4 turns, 2.5 to $5 \mu$ in diameter, Length of spiral is 6 to 20 (occasionally 30 ) $\mu$. Spore: ellipsoid to short rod. 0.7 to 1.2 by 1.0 to $1.4 \mu$ in diameter.

Rarely moist black areas develop on the arial mycelium.

II. Czapek: (A) almost non. (V) colorless. (R) colorless. (P) non. Krainsky: (A) good; brownish white to Light Drab. (R) white to brownish white. (P) non. Bennett: (A) fairly good; white to Pale Mouse Gray or Drab-Gray. (R) brownish yellow to yellowish brown. (P) non. Mod. Bennett (glycerol, fructose and maltose):(A) good; Drab-Gray, Smoke Gray to Light Mouse Gray. (R) yellowish brown. (P) pale brown.

Starch: Hydrolysis negative. Milk: No coagulation; peptonization positive; deep brownish black soluble pigment. Nitrate: Reduction positive.

\section{Streptomyces sp. No. 350}

I. About the same as Streptomyces albus HUT 6047 and Streptomyces $s p$. No. 201, except the growth of long axial aerial mycelium is more prosperous than that of above two strains.

Spiral: sinistrorse, compact (to very compact), 1 to 5 turns, 3 to $6 \mu$ in diameter. Length of spiral is 6 to $37 \mu$. Spore: sphere to ellipsoid, 0.8 to 1.2 by 0.8 to $1.4 \mu$ in diameter.

II. Czapek: (A) good; white to Drab-Gray. (R) pale brownish yellow. (P) almost non. Krainsky: (A) good; Pale Drab-Gray to Drab-Gray. (R) grayish white. (P) non. Bennett: (A) good; white to Pallid Mouse Gray. (R) orangish yellow to yellowish brown. (P) non. Mod. Bennett (glycerol, mannit, starch and maltose): (A) good; Pallid Mouse Gray to Smoke Gray to Drab-Gray. (R) orangish yellow to yellowish brown. (P) non.

Starch: Hydrolysis positive. Milk: No coagulation; peptonization positive; no soluble pigment. Nitrate: Reduction negative. 


\section{Streptomyces sp. No. 202}

I. This strain's long axial aerial mycelium is better developed than that of Streptomyces sp. No. 350 and occasionally shows resemblance to Streptomyces sp. No. 477 as reported previously. Furthermore, a single growth unit of aerial mycelium having no long mycelium is well branched and has resemblance to Streptomyces $s p$. No. $189^{(13)}$ previously reported. These two extremes in morphology are much more conspicuous than in the other strains studied. Of course, the already mentioned general morphological units are large in number. Most terminal hyphae are spiraled, while nonspiraled wavy hyphae are few.

Spiral: sinistrorse, very compact to compact, 2 to 6 turns, 3 to $6 \mu$ in diameter, cylindrical to occasionally snail-like. Length of spiral is 6 to $22 \mu$. Spore: sphere to ellipsoid, 1.0 to 1.4 by 1.0 to $1.6 \mu$.

II. Czapek: (A) fairly good; white to Light Olive-Gray. (V) orange. (R) orange $\rightarrow$ yellowish brown. (P) vivid yellow. Krainsky: (A) good; gray concentrated than Light Drab. (R) yellow $\rightarrow$ yellowish brown. (P) vivid yellow $\rightarrow$ yellowish orange. Bennett: (A) good; Smoke Gray, Light Grayish Olive or Tea Green $\rightarrow$ Light Drab $\rightarrow$ Hair Brown, Benzo Brown. (R) yellow $\rightarrow$ brown. (P) vivid yellow $\rightarrow$ yellowish orange. Mod. Bennett (all 9 c-sources): same as Bennett.

Starch: Hydrolysis positive. Milk: No coagulation; peptonization positive. Nitrate: Reduction negative.

\section{Streptomyces sp. No. 602}

I. Peculiar spirals are observed in this strain. Before sporulation, they are compact, but after sporulation they develop into various shapes, namely from long open to very compact. Furthermore, even in a spiral there is often included various shapes ranging from long open to very compact when sporulated, resulting in random spiral formation.

Spiral: sinistrorse, open to very compact (often random), 4 to 10 turns, 2.5 to $4.5 \mu$ in diameter. Length of spiral is 6 to $40 \mu$. Spore sphere to ellipsoid, 0.6 to 1.0 by 0.8 to $1.6 \mu$ in diameter.

II. Czapek: (A) moderate; white to gray. (V) colorless. (R) brown. (P) brown. Krainsky: (A) moderate; gray. (R) color of aerial mycelium is looked through. (P) non. Bennett: (A) moderate; Drab-Gray to Light Drab. (R) pale yellow to brown. (P) yellowish brown. Mod. Bennett (fructose, maltose and starch):(A) good; gray concentrated than Light Drab or intermediate color between Drab and Hair Brown. (R) pale yellow to dark brown. (P) almost non.

Starch: Hydrolysis positive. Milk: No coagulation; peptonization positive. Nitrate: Reduction negative.

Streptomyces sp. No. 469

I. The morphological features are in conformity with general morphology. Spiral: sinistrorse, compact (occasionally very compact), 2 to 8 turns, 3 
to $4 \mu$ in diameter. Length of spiral is 4 to $18 \mu$. Spore: ellipsoid to short rod, 0.7 to 1.0 by 0.8 to $1.6 \mu$ in diameter.

II. Czapek: growth weak. (A) non. (V) colorless. (R) colorless. (P) non. Krainsky: (A) good; (Pale or) Light Vinaceous-Lilac to Pale VinaceousDrab. (R) dark reddish violet. (P) brown. Bennett: (A) good; white $\rightarrow$ purplish white $\rightarrow$ Drab-Gray $\rightarrow$ Benzo Brown $\rightarrow$ Blackish Brown $(I)$. (R) dark reddish violet. (P) brown. Mod. Bennett (all 9 c-sources): (A) good; white $\rightarrow$ pale reddish purple $\rightarrow$ Drab-Gray $\rightarrow$ Benzo Brown, Fuscous, Fuscous-Black or Blackish Brown (I) when self-moistened. (R) dark reddish violet. (P) brown.

Starch: Hydrolysis positive. Milk: No coagulation; no peptonization; brownish gray soluble pigment. Nitrate: Reduction positive.

\section{Streptomyces sp. No. 203}

I. This strain's side branches are gigantic and often difficult to distinguish them from axial parts. Spirals are also gigantic.

Spiral: sinistrorse, open to compact (sometimes open to compact parts are contained in a spiral), 2 to 6 turns, 3 to $9 \mu$ in diameter. Length of spiral is 15 to $100 \mu$. Spore: ellipsoid to short rod to a little long rod, 0.6 to 1.0 by 1.0 to $1.6 \mu$ in diameter.

II. Czapek: (A) moderate; white. (R) pale yellow to orange. (P) almost non. Krainsky: (A) moderate; white. (R) white. (P) non. Bennett: (A) slight to moderate; almost white. (R) pale orange. $(\mathrm{P})$ pale yellow. Mod. Bennett (mannit, sucrose, maltose and starch): (A) good; Shell Pink, Pale Vinaceous-Pink to Pale Grayish Vinaceous. (R) yellow to brown. (P) yellow to yellowish brown.

Starch: Hydrolysis negative. Milk: No coagulation; peptonization negative or very weak; brownish gray soluble pigment. Nitrate: Reduction negative.

\section{SUMMARY}

The microscopic morphology of Streptomyces hygroscopicus and morphologically related strains are described. These strains have sinistrorse spirals in terminal filaments of side branches arisen from axial mycelia. Axial mycelia are fairly long but sometimes short, straight to flexous, and the branching system principally takes various monopodial forms in its early stage of growth, but often becomes to appear irregular after full growth has been attained. Side branches range from single to branched.

Description of each strain employed is also given.

The author wishes to express his sincere thanks to Dr. K. SaKaguchi for his guidance and to Dr. T. Nehira, Dr. K. Arima and Dr. Y. Ikeda for their encouragement. The author is indebted to Mr. T. Hasegawa and Dr. H. IIzuka for their kindness in supplying strains used in this study. This work was supported in part by Grant for Co-Operative Research from the 
Ministry of Education and in part by Research Subsidy from WaKSmaN Foundation in Japan.

\section{REFERENCES}

(1) C. DrechSler: Bot. Gaz., 67, 65, 147 (1919).

(2) S. A. Waksman and H. A. LeChevalier: Guide to the Classification and Identification of the Actinomycetes and their Antibioticus (1953).

(3) N. A. Krassilnikov: Acad. Soci., USSR, Moscow (1949) (translated into English by J. B. Routien, 1957).

(4) E. BaldacCI, C. Spalla and A. Grein: Arch. f. Mikrobiol., 20, 347 (1954).

(5) T. G. Pridham, C. W. Hesseltine and R. G. Benedict: Appl. Microbiol., 6, 52 (1958).

(6) L. Ettlinger, R. Corbaz und R. Huetter: Arch. f. Mikrobiol., 31, 326 (1958).

(7) H. D. Tresner and E. J. Backus: Appl. Microbiol., 4, 243 (1956).

(8) H. NAGANiSHI and R. Nomi: J. Ferment. Techn. Japan, 32, 406 (1954).

(9) ibid., 32, 434 (1954).

(10) ibid., 32, 492 (1954).

(11) ibid., 33, 13 (1955).

(12) ibid., 33, 167 (1955).

(13) ibid., 33, 209 (1955).

(14) ibid., 33, 294 (1955).

(15) R. Nomi: ibid., 33, 486 (1955).

(16) H. Naganishi and R. Nomi: J. Ferment. Techn. Japan, 31, 476 (1953); R. NomI: ibid., 33, 341 (1955). 


\section{EXPLANATION OF PLATES I-III}

Fig. 1 and Photos. 1-4 are S. hygroscopicus HUT 6026. Fig. 1 is the sketch. Photo. 1 (photographed with $100 \times$ microscopic magnification) shows a part of colony. Photo. 2 (photographed with $450 \times$ ) shows appearance of short side branches arising from axial filaments. Photo. $3(450 \times)$ shows appearance of a part of colony bearing thickly branched side branches. Photo. $4(1000 \times)$ shows very compact spirals.

Photos. 5-7 are S. violaceoniger HUT 6088. Photo. $5(100 \times)$ shows a part of colony. Photo. $6(450 \times)$ shows appearance of short side branches arising from axial filaments. Photo. $7(450 \times)$ shows a part of colony bearing thickly branched side branches.

Photos. 8-10 are S. albus HUT 6047 . Photo. $8(100 \times)$ shows appearance of branching axial mycelia where short side branches are not yet borne. Photo. $9(100 \times)$ shows appearance of a part of colony where axial mycelia have already borne many side branches with spirals. Photo. $10(450 \times)$ shows a part of colony where short side branches are attached to axial mycelia. The short side branches are longer than those of the above two strains.

Photo. $11(450 \times)$ is Streptomyces sp. No. 201 showing a part of colony where short side branches are attached to flexous axial mycelia.

Photos. 12-14 are Streptomyces sp. No. 350. Photo. $12(100 \times)$ shows a part of colony in which are seen young growth unit having a few side branches as well as well developed growth unit having fairly many side branches. Photo. $13(450 \times)$ shows side branches attached to flexous axial mycelia. Photo. $14(450 \times)$ shows thickly branched side branches attached to axial mycelia.

Photos. 15-19 are Streptomyces sp. No. 202. Photo. $15(100 \times)$ shows a colonial part with a little flexous axial mycelia bearing side branches. Photo. $16(100 \times)$ shows a colonial part with straight axial mycelia bearing side branches. Photo. $17(100 \times)$ shows growth units which have no long axial mycelia but the morphology resembles S. coelicolor-type. Photo. $18(450 \times)$ shows side branches attached to axial mycelia. Photo. $19(450 \times)$ shows a coelicolor-type's growth unit.

Photos. 20-23 are Streptomyces sp. No. 602. Photo. $20(100 \times)$ shows a part of colony. Photo. $21(450 \times)$ shows side branches attached to axial mycelia. Photo. 22 $(450 \times)$ shows a simple growth unit which is not a side branch, while Photo. $23(450 \times)$ shows a well developed side branch, and in those two photographs various forms of spirals are observed.

Photos. 24-26 are Streptomyces sp. No. 469. Photos. 24 and 25 (both $100 \times$ ) show appearance of a part of colony where various growth stages are seen which range from axial mycelia bearing no side branches to those bearing many side branches. Photo. $26(450 \times)$ shows side branches attached to axial mycelia.

Photos. 27-30 are Streptomyces sp. No. 203. Photo. $27(100 \times)$ shows a part of colony and Photo. $28(100 \times)$ shows a growth unit. Photos. 29 and 30 (both $450 \times$ ) show side branches attached to axial mycelia. Since the side branches in this strain are gigantic, it is not easy to contain side branches and axial mycelia together in one photograph.

One unit of the scale in the photographs is $11 \mu$ in $100 \times$ 's photographs, $2.5 \mu$ in $450 \times$ 's photographs and $1.1 \mu$ in $1000 \times$ 's photographs. 

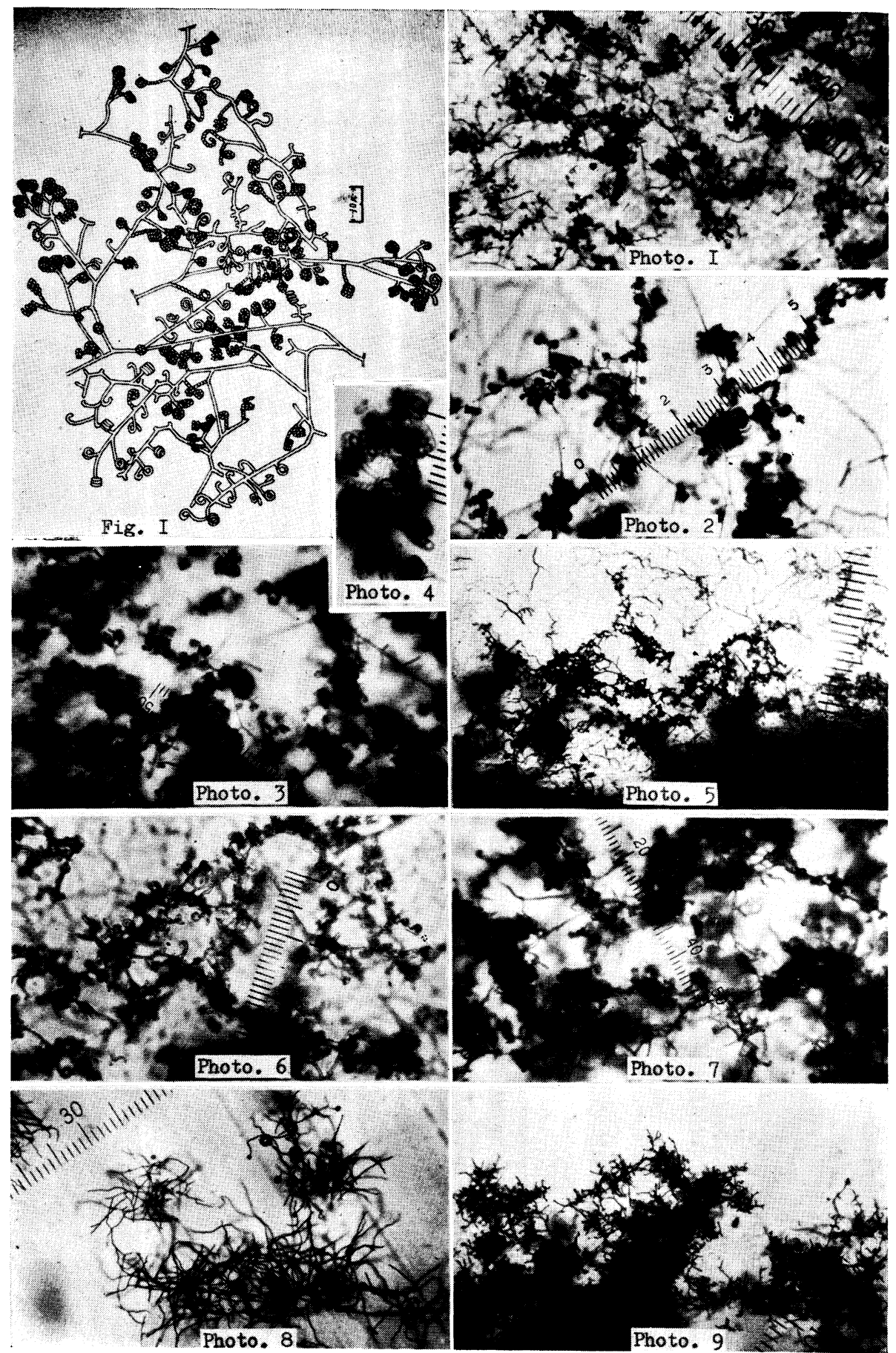


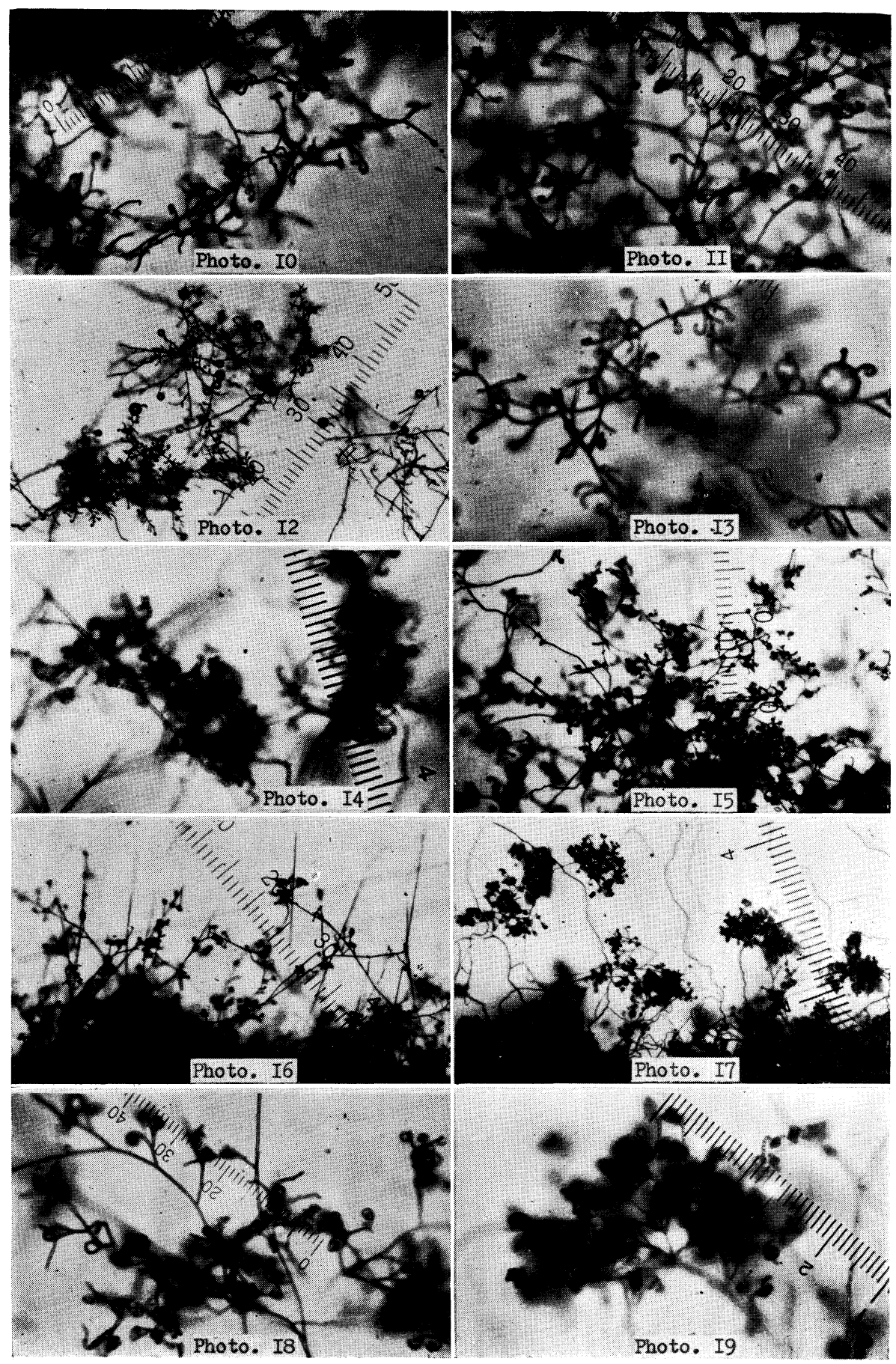




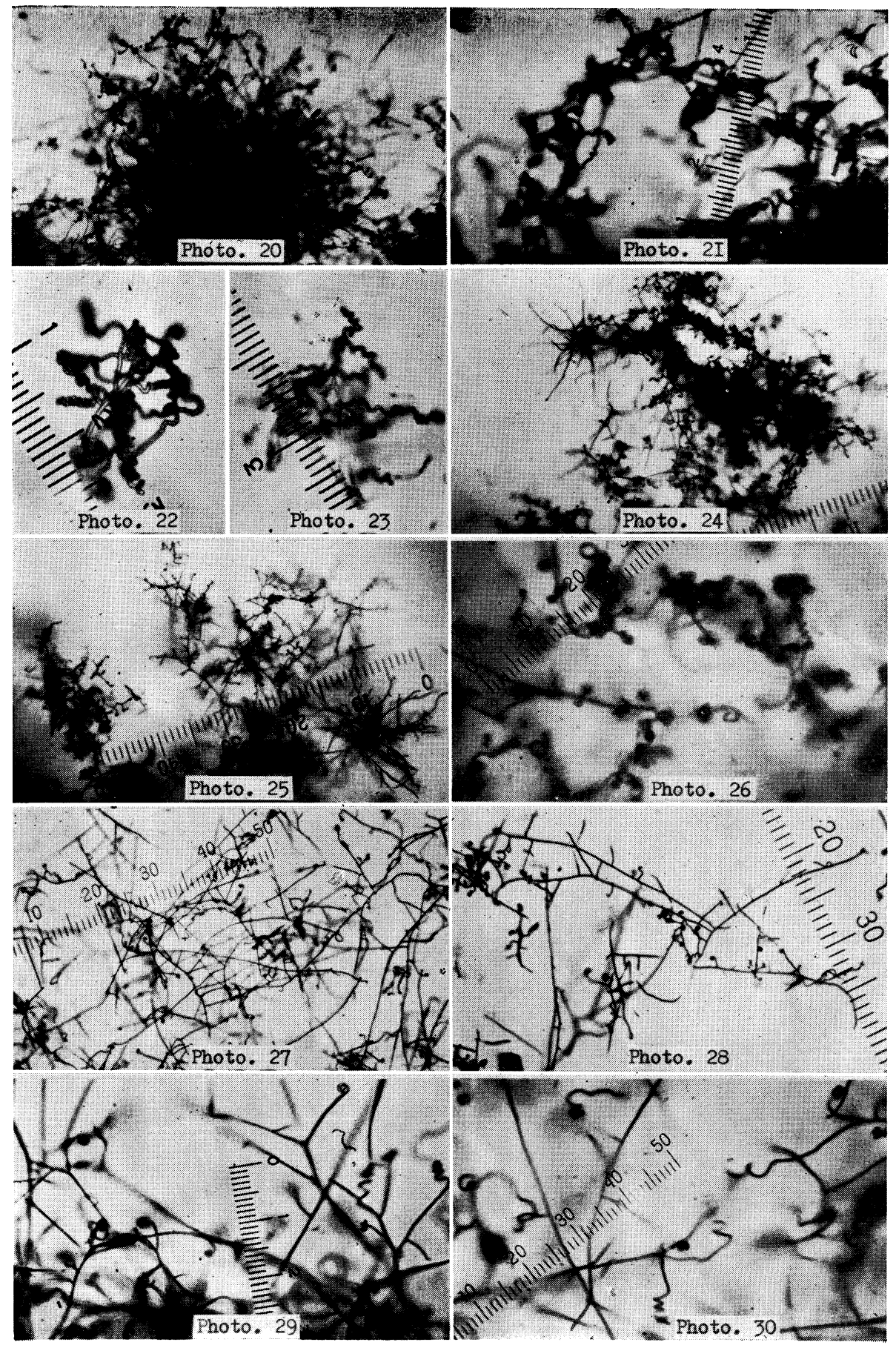

